\title{
Imaging surveillance for surgically resected stage I non-small cell lung cancer: Is more always better?
}

\author{
Melanie Subramanian, MD, ${ }^{\mathrm{a}}$ Jingxia Liu, PhD, ${ }^{\mathrm{b}}$ Caprice Greenberg, MD, MPH, \\ Jessica Schumacher, PhD, MS, ${ }^{\mathrm{c}}$ George J. Chang, MD, MS, ${ }^{\mathrm{d}}$ Timothy L. McMurry, PhD, ${ }^{\mathrm{e}}$ \\ Amanda B. Francescatti, MS, ${ }^{f}$ Tara R. Semenkovich, MD, MPHS, ${ }^{a}$ Jessica L. Hudson, MD, MPHS, ${ }^{a}$ \\ Bryan F. Meyers, MD, MPH, ${ }^{a}$ Varun Puri, MD, MSCI, ${ }^{\mathrm{a}}$ and Benjamin D. Kozower, MD, $\mathrm{MPH}^{\mathrm{a}}$
}

\section{ABSTRACT}

Objective: Routine surveillance imaging for patients with resected non-small cell lung cancer is standard for the detection of disease recurrence and new primary lung cancers. However, surveillance intensity varies widely in practice, and its influence on long-term outcomes is poorly understood. We hypothesized that surveillance intensity was not associated with 5-year overall survival in patients with resected stage I non-small cell lung cancer. Additionally, we examined patterns of recurrence and new primary lung cancer development.

Methods: Cancer registrars at the Commission on Cancer accredited institutions re-abstracted records to augment National Cancer Database patient data with information on comorbidities, imaging surveillance including intent and result of imaging, and recurrence (2007-2012). Pathologic stage I non-small cell lung cancer patients undergoing computed tomography surveillance were placed into 3 imaging surveillance groups based on clinical practice guidelines: high intensity (3 month), moderate intensity (6 month), and low intensity (annual). KaplanMeier analysis and Cox regression were used to compare overall survival among the 3 surveillance groups.

Results: Two thousand four hundred forty-two patients were identified, with 805 $(33 \%), 1216(50 \%)$, and $421(17 \%)$ patients in the high, moderate, and low surveillance intensity groups, respectively. Five-year overall survival was similar between intensity groups $(P=.547)$. Surveillance on asymptomatic patients detected $210(63 \%)$ cases of locoregional recurrences and $128(72 \%)$ cases of new primary lung cancer.

Conclusions: In a unique national dataset of long-term outcomes for stage I nonsmall cell lung cancer, surveillance intensity was not associated with 5-year overall survival. (J Thorac Cardiovasc Surg 2019;157:1205-17)

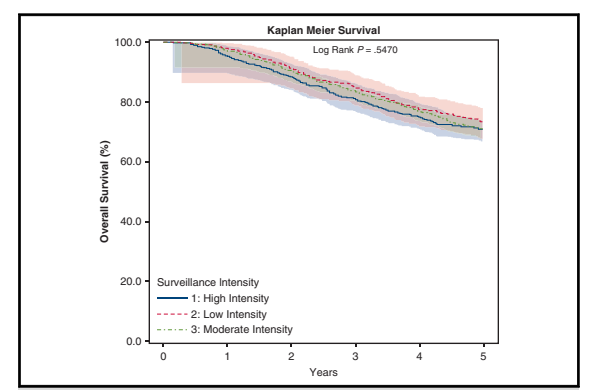

Patients receiving different intensities of $\mathrm{CT}$ surveillance have similar overall survival.

\section{Central Message}

Surveillance imaging intensity after resection for pathologic stage I non-small cell lung cancer is not associated with long-term overall survival.

\section{Perspective}

There are limited quality long-term data on postoperative imaging surveillance for patients with resected non-small cell lung cancer. Using a large, nationally representative database containing longitudinal data on surgically resected pathologic stage I non-small cell lung cancer patients, we compared 5-year overall survival among patients undergoing different imaging surveillance intensities.

See Commentary on page 1218.

See Editorial page 1194.
From the Divisions of ${ }^{\mathrm{a}}$ Cardiothoracic Surgery and ${ }^{\mathrm{b}}$ Public Health Sciences, Washington University School of Medicine, St Louis, Mo; ${ }^{c}$ Department of Surgery, School of Medicine and Public Health, University of Wisconsin, Madison, Wis; ${ }^{\mathrm{d}}$ Department of Surgical Oncology, University of Texas MD Anderson Cancer Center, Houston, Tex; ${ }^{\mathrm{e}}$ Department of Public Health Sciences, University of Virginia School of Medicine, Charlottesville, Va; and ${ }^{\mathrm{f}}$ American College of Surgeons, Chicago, Ill.

Supported by the Patient-Centered Outcomes Research Institute (grant No. R-APD1306-00727 to Dr Kozower). Dr Subramanian is supported by T32 National Institutes of Health Cardiothoracic Training Grant No. 5T32HL007776023.

Read at the 44th Annual Meeting of the Western Thoracic Surgical Association, Goleta, California, June 27-30, 2018.

Received for publication June 26, 2018; revisions received Sept 12, 2018; accepted for publication Sept 19, 2018.

Address for reprints: Benjamin D. Kozower, MD, MPH, 660 S. Euclid Ave, Campus Box 8234, St Louis, MO 63110 (E-mail: kozowerb@wustl.edu). $0022-5223 / \$ 36.00$

Copyright $\odot 2018$ by The American Association for Thoracic Surgery

https://doi.org/10.1016/j.jtcvs.2018.09.119
Surveillance after lung resection remains a critical component of survivorship care for patients with stage I non-small cell lung cancer (NSCLC). ${ }^{1,2}$ Surveillance imaging can allow for the early detection of recurrence or screening for new primary lung cancer (NPLC). ${ }^{3,4}$ For patients whose recurrence or NPLC is detected early, there is a

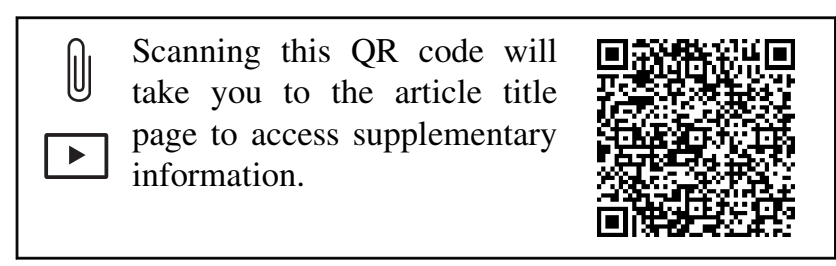




\section{Abbreviations and Acronyms \\ $\mathrm{CoC}=$ Commission on Cancer \\ $\mathrm{CT}=$ computed tomography \\ NCDB $=$ National Cancer Database \\ NPLC $=$ new primary lung cancer \\ NSCLC $=$ non-small cell lung cancer \\ OS $\quad=$ Overall survival}

possibility that additional curative intent therapy can be performed. ${ }^{3}$

Recommendations regarding surveillance imaging intensity after NSCLC resection vary widely in clinical practice. Existing current guidelines recommend visits as frequent as every 3 months to annual visits during the first 2 years. ${ }^{5-7}$ The influence of surveillance imaging on long-term survival is poorly understood. The lack of consensus is due to limited quality longitudinal follow-up data on postresection patients. $^{3,8}$

We received support from the Patient Centered Outcomes Research Institute to study postresection cancer surveillance. Specifically, we planned 2 separate analyses focusing on the utility of imaging surveillance after resection of NSCLC. McMurry and colleagues ${ }^{9}$ published the first analysis, which studied the influence of imaging surveillance intensity on overall and postrecurrence survival in surgically resected stage I to III NSCLC. Given anticipated stagespecific differences in surveillance practices, recurrence risk and survival, and the paucity of published stagespecific surveillance data, we performed a second planned analysis using independent study design and statistical methodology to examine imaging surveillance intensity in resected stage I NSCLC patients. Additionally, we describe the risk of development of recurrence and NPLC, method of detection of recurrent tumors, and postrecurrence treatment for this population.

We performed a retrospective cohort study using supplemented data from the National Cancer Database (NCDB) to compare imaging surveillance in patients with pathologic stage I NSCLC. Our primary aim was to determine whether the intensity of surveillance with computed tomography (CT) was associated with 5-year overall survival (OS). We hypothesized that surveillance intensity was not associated with OS. Additionally, we performed descriptive analyses of disease recurrence (locoregional and distant), and development of NPLC.

\section{METHODS \\ Study Population and Cohort Selection}

Using the special study mechanism (described in the next section), we identified patients who underwent resection for pathologic stage I NSCLC. Patients were required to have undergone postoperative imaging surveillance. We only analyzed CT scans (as opposed to chest radiographs) based on published level I evidence advocating for CT imaging as standard of care in lung cancer screening. ${ }^{10}$ The National Lung Screening Trial randomized high-risk patients to receive low-dose CTs or standard chest radiograph. $^{10,11}$ The trial found that CT scanning was associated with significantly higher detection of NSCLC and lower mortality. Given this level I evidence suggesting the superiority of CT imaging, only CT scans were considered for analyses. We a priori constructed surveillance intensity cohorts (high intensity, moderate intensity, and low intensity) based on current guidelines. We created visit windows to assign patients into these cohorts based on days from surgery to first surveillance CT. These visit windows included 60 to 150 days ( 3 months), 151 to 300 days (6 months), and 301 to 450 days (1 year) which corresponded to the high-, moderate-, and low-intensity cohorts, respectively.

\section{Data Abstraction}

To obtain the detailed level of information required for this study, registrars at Commission on Cancer $(\mathrm{CoC})$ accredited institutions abstracted comorbidity, surveillance, and cancer recurrence/NPLC information utilizing the special study mechanism of the $\mathrm{CoC}$, which supplemented data that already reside in the NCDB.

Up to 10 NSCLC patients were randomly selected from each institution for further abstraction. These patients underwent surgery for clinical stage I to III NSCLC (January 2006-December 2007), were alive 90 days postsurgery, and had available medical records. Patients with unknown recurrence status were excluded without replacement from the same institution. Patients were followed for 5 years postdiagnosis or until death.

Registrars obtained information on comorbidities, locoregional and distant recurrence, and development of NPLC for 5 years following surgery. Differentiation between disease recurrence and NPLC was based on original documentation by the treating clinician in the medical record. Locoregional recurrence was defined as tumor recurrence in the ipsilateral and/or regional lymph nodes, whereas distant recurrence was defined as tumor recurrence in the contralateral lung/lymph nodes or nonlung site.

Detailed information on postoperative imaging, date, and indication for imaging were recorded. Indications included surveillance in absence of a new sign/symptom, follow-up for new sign/symptom, follow-up for suspicious finding on other imaging, imaging performed in response to newly detected malignancy, not cancer related, or unable to determine. Registrars used radiology reports as the primary source to obtain symptom status. They also had access to outpatient records, including clinic and consult notes from visits that preceded the scan and made mention of intent to order imaging as a consequence of a new sign or symptom. These notes were from primary care providers, medical oncologists, radiation oncologists, surgeons, or other relevant providers. When available, information was collected on whether or not a patient underwent treatment (eg, surgery, chemotherapy, radiation, or combination therapy) for recurrent disease. Registrars were trained by weekly webinars to standardize the abstraction process. Study data were merged with existing NCDB data by the CoC, deidentified, and provided to the study team. Data collection was completed in 2015. Data were deemed not human subjects research and were exempted from institutional review board review.

\section{Inclusion and Exclusion Criteria}

We restricted our study inclusion to patients with pathologic stage I cancer who underwent surgical resection and had their first surveillance imaging CT scan between 60 and 450 days after surgical resection. Additionally, patients must have been asymptomatic at the time of the first postoperative CT. We excluded patients who underwent chemoradiation therapy, had positive surgical margins, or for whom the indication for first surveillance CT was unknown (Figure E1).

\section{Descriptive and Inferential Statistics}

Descriptive statistics were reported using mean \pm standard deviation for those variables that were approximately normally distributed, median 
(quartile 1-quartile 3) for highly skewed variables, and count (\%) for categorical variables. Continuous variables were compared between the 3 cohorts using Kruskal-Wallis tests, whereas categorical data were assessed with $\chi^{2}$ or Fisher exact tests where appropriate. All $P$ values were 2 -tailed. For each treatment cohort, the median number of asymptomatic surveillance images per person-year were calculated. Person-years were defined as follow-up time until death, loss to follow-up, or first onset of recurrence/NPLC. Median numbers of surveillance images were reported for 2 person-years because the majority of existing practice guidelines are framed within the first 2 years of patient follow-up. ${ }^{5-7}$
Cumulative incidences of locoregional/distant recurrence were estimated using competing risks analysis, with death or other recurrence/ NPLC treated as competing risks. Covariates included in the disease recurrence competing risk models were determined a priori based on clinical experience and literature review and included pathologic stage, lymph nodes sampled, tumor size, tumor histology, tumor grade, and surgical procedure. The final model included the variables with $P<.10$, where group, pathologic stage, and number of lymph nodes were forced into the model. Overall survival for all 3 cohorts was modeled using univariate and multivariable Cox proportional hazards regression analysis, and

TABLE 1. Patient and tumor-related factors, all patients

\begin{tabular}{|c|c|c|c|c|c|}
\hline Variable & $\begin{array}{l}\text { All patients } \\
(\mathrm{N}=\mathbf{2 4 4 2})\end{array}$ & $\begin{array}{l}\text { High intensity } \\
\quad(\mathbf{n}=\mathbf{8 0 5})\end{array}$ & $\begin{array}{l}\text { Moderate intensity } \\
\quad(n=1216)\end{array}$ & $\begin{array}{l}\text { Low intensity } \\
\quad(n=421)\end{array}$ & $P$ value \\
\hline \multicolumn{6}{|l|}{ Patient-related factors } \\
\hline Age (y) & $66.2(9.7)$ & $65.7(9.9)$ & $66.4(9.7)$ & $66.6(9.6)$ & .171 \\
\hline Male sex & $1106(45.3)$ & $340(42.3)$ & $569(46.8)$ & $197(46.8)$ & .110 \\
\hline Race & & & & & .698 \\
\hline White & $2198(90.0)$ & $729(90.6)$ & $1096(90.1)$ & 373 (88.6) & \\
\hline African American & $176(7.2)$ & $56(7.0)$ & $88(7.3)$ & $32(7.6)$ & \\
\hline Other & $68(2.8)$ & $20(2.4)$ & $32(2.6)$ & $16(3.8)$ & \\
\hline Charlson/Deyo score & & & & & .381 \\
\hline 0 & $1236(50.6)$ & $414(51.4)$ & $622(51.2)$ & $200(47.5)$ & \\
\hline 1 & $890(36.4)$ & $278(34.5)$ & $445(36.6)$ & 167 (39.7) & \\
\hline $2+$ & $316(13.0)$ & $113(14.1)$ & $149(12.2)$ & $54(12.8)$ & \\
\hline \multicolumn{6}{|l|}{ Comorbidity } \\
\hline Chronic obstructive pulmonary disease & 998 (40.9) & $324(40.3)$ & 485 (39.9) & $189(44.9)$ & .179 \\
\hline Congestive heart failure & $131(5.4)$ & $50(6.2)$ & $60(4.9)$ & $21(5.0)$ & .428 \\
\hline Coronary artery disease & $531(21.7)$ & $168(20.9)$ & $260(21.4)$ & $103(24.5)$ & .319 \\
\hline Diabetes & $357(14.6)$ & $129(16.0)$ & $170(14.0)$ & $58(13.8)$ & .385 \\
\hline Peripheral vascular disease & $224(9.2)$ & $64(8.0)$ & $109(9.0)$ & $51(12.1)$ & .053 \\
\hline Psychiatric history & $197(8.1)$ & $76(9.4)$ & $88(7.2)$ & $33(7.8)$ & .201 \\
\hline Substance abuse & $124(5.1)$ & $46(5.7)$ & $56(4.6)$ & $22(5.2)$ & .533 \\
\hline \multicolumn{6}{|l|}{ Tumor-related factors } \\
\hline Surgical resection & & & & & .478 \\
\hline Wedge resection & $353(14.4)$ & $105(13.0)$ & $183(15.1)$ & $65(15.5)$ & \\
\hline Segmentectomy & $65(2.7)$ & $28(3.5)$ & $26(2.1)$ & $11(2.6)$ & \\
\hline Lobectomy/bilobectomy & $1980(81.1)$ & $659(81.9)$ & $985(81.0)$ & $336(79.8)$ & \\
\hline Pneumonectomy & $44(1.8)$ & $13(1.6)$ & $22(1.8)$ & $9(2.1)$ & \\
\hline Tumor size (mm) & $22(15-30)$ & $22(15-30)$ & $22(15-30)$ & $21(15-30)$ & .819 \\
\hline No. of lymph nodes sampled & $1(1-1)$ & $1(1-1)$ & $1(1-1)$ & $1(1-1)$ & .278 \\
\hline Pathologic stage & & & & & .904 \\
\hline $1 \mathrm{~A}$ & $1497(61.3)$ & $485(60.2)$ & $754(62.0)$ & $258(61.3)$ & \\
\hline $1 \mathrm{~B}$ & $866(35.5)$ & $292(36.3)$ & $426(35.0)$ & $148(35.1)$ & \\
\hline 1-A/B unknown & $79(3.2)$ & $28(3.5)$ & $36(3.0)$ & $15(3.6)$ & \\
\hline Histology & & & & & .935 \\
\hline Adenocarcinoma & $1161(47.6)$ & $391(48.6)$ & $572(47.2)$ & $198(47.0)$ & \\
\hline Squamous & $647(26.5)$ & $209(26.0)$ & $324(26.7)$ & $114(27.1)$ & \\
\hline Other & $489(20.1)$ & $159(19.7)$ & $241(19.9)$ & $89(21.1)$ & \\
\hline Unknown & $141(5.8)$ & $46(5.7)$ & $75(6.2)$ & $20(4.8)$ & \\
\hline Grade & & & & & .567 \\
\hline Well differentiated & $402(16.5)$ & $136(16.9)$ & $195(16.0)$ & $71(16.9)$ & \\
\hline Moderately differentiated & $1071(43.9)$ & $345(42.9)$ & $540(44.4)$ & $186(44.1)$ & \\
\hline Poorly differentiated & $765(31.3)$ & $251(31.2)$ & $379(31.2)$ & $135(32.1)$ & \\
\hline Undifferentiated & $18(0.7)$ & $8(1.0)$ & $5(0.4)$ & $5(1.2)$ & \\
\hline Indeterminate & $186(7.6)$ & $65(8.0)$ & $97(8.0)$ & $24(5.7)$ & \\
\hline
\end{tabular}

Values are presented as $\mathrm{n}(\%)$ or median (interquartile range). 
covariate effects are presented as hazard ratios with $95 \%$ confidence ratios. The proportionality assumption was tested by adding a time-dependent covariate for each variable. When the test indicated nonproportional hazards over time, models were divided into 2 time periods, and the maximized partial likelihood method was used to find the most appropriate breakpoint and the proportionality assumptions were further tested. Interested covariates in Cox proportional hazard modeling were also determined a priori based on literature and clinical significance and included age, gender, race, surveillance intensity group, chronic obstructive pulmonary disease, congestive heart failure, coronary artery disease, diabetes, psychiatric disease, pathologic stage, tumor size, tumor grade, lymph nodes sampled, tumor histology, and surgical procedure. The final model was built through the stepwise selection, where group, pathologic stage, and number of lymph nodes were forced into the model. Kaplan-Meier survival curves were also constructed to compare OS among surveillance cohorts.

Missing covariate data were $\leq 1 \%$ in all demographic variables: 4 patients were missing tumor histology, and 1 patient was missing gender status. Given the low number of patients with missing data, missing covariate information was reported in descriptive statistics, but was not included in regression analyses. Data that were reported as unknown or indeterminate were handled as separate categorical variables for analyses. All analyses were performed using SAS version 9.4 (SAS Institute Inc, Cary, NC).

\section{RESULTS}

We identified 4340 patients with pathologic stage I NSCLC who underwent postoperative CT imaging. When examining indication for the first CT scan, 3170 patients $(73 \%)$ were asymptomatic and eligible for inclusion as true surveillance imaging. Two thousand five hundred sixty-five patients received their first scan within 60 to 450 days from surgery. We excluded 123 patients who underwent chemoradiation therapy or had positive surgical margins, leaving 2442 patients in our study cohort that met inclusion criteria. Of included patients, $805(33 \%)$, $1216(50 \%)$, and $421(17 \%)$ patients were placed in the high-intensity, moderate-intensity, and low-intensity cohorts, respectively (Figure E1). When calculating the median number of asymptomatic surveillance images, highintensity, moderate-intensity, and low-intensity groups received 4.67 (median Q1-Q3, 2.43-15.02), 3.65 (median Q1-Q3, 1.97-12.54), and 2.96 (median Q1-Q3, 1.7110.27) images per 2 person-years, respectively. This confirmed that our predefined surveillance intensity cohorts, which were based on time from surgery to time to first surveillance scan, corresponded to differing frequencies of imaging. The cohorts demonstrated similarity across most patient, tumor, and treatment-related variables (Table 1).

\section{Surveillance Intensity and Survival Analysis}

Cox proportional hazard modeling showed that surveillance intensity was not associated with OS $(P=.302)$ (Table 2). Factors associated with worse overall survival included age, pathologic stage, male gender, chronic obstructive pulmonary disease, congestive heart failure, psychiatric disease, histologic grade, and nonlobectomy surgical resection $(P<.05)$. Kaplan-Meier analysis also showed similar 5-year OS probability among our
TABLE 2. Cox proportional hazard model of 5-year overall survival

\begin{tabular}{|c|c|c|}
\hline Covariate & $\begin{array}{l}\text { Hazard ratio }(95 \% \\
\text { confidence interval) }\end{array}$ & $P$ value \\
\hline Age & $1.03(1.02-1.04)$ & $<.001$ \\
\hline \multicolumn{3}{|l|}{ Gender } \\
\hline Male & Reference & .036 \\
\hline Female & $0.85(0.73-0.99)$ & \\
\hline \multicolumn{3}{|l|}{ Comorbidity } \\
\hline $\begin{array}{l}\text { Chronic obstructive } \\
\text { pulmonary disease }\end{array}$ & $1.27(1.09-1.48)$ & .003 \\
\hline Congestive heart failure & $1.57(1.20-2.05)$ & .001 \\
\hline Psychiatric history & $1.54(1.20-1.98)$ & .001 \\
\hline \multicolumn{3}{|l|}{ Pathologic stage } \\
\hline $1 \mathrm{~A}$ & Reference & .024 \\
\hline 1B & $1.25(1.06-1.46)$ & \\
\hline 1 & $1.11(0.73-1.70)$ & \\
\hline \multicolumn{3}{|l|}{ Histologic grade } \\
\hline Well differentiated & Reference & $<.001$ \\
\hline Moderately differentiated & $1.65(1.26-2.16)$ & \\
\hline Poorly differentiated & $1.76(1.32-2.35)$ & \\
\hline Undifferentiated & $4.02(2.03-7.97)$ & \\
\hline Unknown & $1.62(1.11-2.36)$ & \\
\hline \multicolumn{3}{|l|}{ Resection type } \\
\hline Lobectomy/bilobectomy & Reference & .015 \\
\hline Wedge resection & $1.32(1.07-1.63)$ & \\
\hline Segmentectomy & $1.39(0.93-2.09)$ & \\
\hline Pneumonectomy & $1.48(0.89-2.46)$ & \\
\hline $\begin{array}{l}\text { Number of lymph nodes } \\
\text { sampled }\end{array}$ & $0.97(0.84-1.12)$ & .689 \\
\hline Surveillance group & & .302 \\
\hline Low intensity & Reference & \\
\hline Moderate intensity & $1.13(0.91-1.39)$ & \\
\hline High intensity & $1.20(0.95-1.50)$ & \\
\hline
\end{tabular}

surveillance intensity cohorts $(70.7 \%$ high intensity vs $70.9 \%$ moderate intensity vs $73.2 \%$ low intensity; $P=.547$ ) (Figure 1).

\section{Locoregional Disease Recurrence}

Five-year cumulative incidence of locoregional recurrence was similar across surveillance intensity cohorts (high intensity, 0.13 ; 95\% confidence interval [CI], 0.110.15 ; moderate intensity, 0.13 ; $95 \% \mathrm{CI}, 0.11-0.15$; and low intensity, 0.14; 95\% CI, 0.11-0.17 $[P=.967])$ (Figure 2). Variables associated with increased risk of locoregional disease recurrence included tumor size, histology, and nonlobectomy resection type $(P<.05)$ (Table 3$)$. Of those with locoregional disease recurrence, 166 (49.9\%), $96(28.8 \%)$, and $66(19.8 \%)$ had evidence of locoregional disease in the same lung, regional lymph nodes, or both lung and lymph nodes, respectively. For 5 patients $(1.5 \%)$, the location of local recurrence was unable to be determined. When determining how local recurrences 


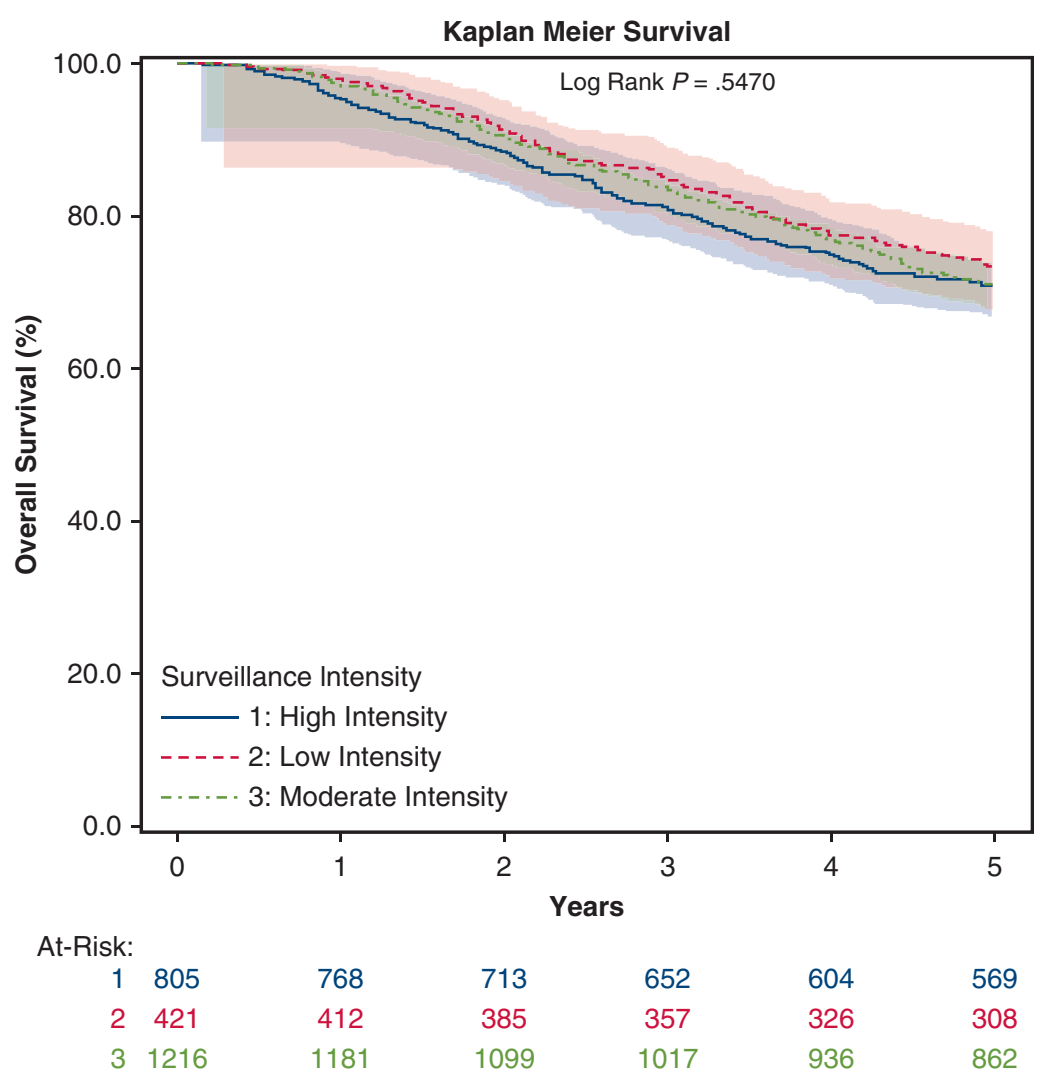

FIGURE 1. Overall survival by surveillance intensity group.

were first detected, $210(63.1 \%)$ patients had disease first detected by routine surveillance CT scans and were asymptomatic at the time of imaging (Table E1).

Of those who had locoregional recurrence, 243 patients $(73.0 \%)$ underwent subsequent treatment after detection of locoregional recurrence (Video 1). Of those who did not undergo subsequent treatment, 24 patients $(21.8 \%)$, 30 patients $(18.4 \%)$, and 16 patients $(26.7 \%)$ belonged to the high-intensity, moderate-intensity, and low-intensity groups, respectively.

\section{Distant Recurrence}

Five-year cumulative incidence of distant recurrence was significantly difference across surveillance intensity groups (high intensity, 0.15; 95\% CI, 0.13-0.18; moderate intensity, 0.13; 95\% CI, 0.11-0.15; and low intensity, 0.10; $95 \%$ CI, 0.07-0.13 [P=.024]) (Figure 3). Variables associated with distant recurrence included surveillance intensity, tumor size, tumor grade, and histology $(P<.05)$ (Table 4$)$. Bone metastases were the most common site of distant recurrence in 89 patients $(26.6 \%)$, followed by contralateral lung and brain metastases in 87 patients $(26.0 \%)$ and 76 patients $(22.8 \%)$, respectively. Routine surveillance imaging detected distant recurrence in 122 patients $(36.6 \%)$, whereas 135 patients $(40.5 \%)$ had recurrence detected by imaging ordered after new signs or symptoms developed (Table E1). Of those with distant recurrence, 83 patients $(24.9 \%)$ did not undergo any treatment (Table E2), with 24 patients $(19.4 \%), 43$ patients $(26.4 \%)$, and 16 patients $(34.8 \%)$ belonging to the high-intensity, moderate-intensity, and low-intensity groups, respectively.

\section{Development of NPLC}

Five-year cumulative incidence of NPLC was similar across surveillance intensity groups (high intensity, 0.05 ; 95\% CI, 0.04-0.08; moderate intensity, $0.08 ; 95 \% \mathrm{CI}$, 0.06-0.11; and low intensity, 0.07 ; $95 \%$ CI, $0.06-0.09$ $[P=.393])$. Ninety-seven patients $(54.5 \%)$ developed an NPLC with lung cancer histology different from their initial tumor. Routine surveillance imaging detected NPLC in 128 cases $(71.9 \%)$ (Table E1).

\section{CONCLUSIONS}

There are more than 400,000 lung cancer survivors in the United States. ${ }^{12}$ These individuals are at high risk for developing recurrence or NPLC. ${ }^{1,12}$ Despite the need for evidence-based surveillance guidelines, existing guidelines are not uniform and are based on small retrospective studies and expert opinion. Organizations, including the American College of Chest Physicians, the National Comprehensive 


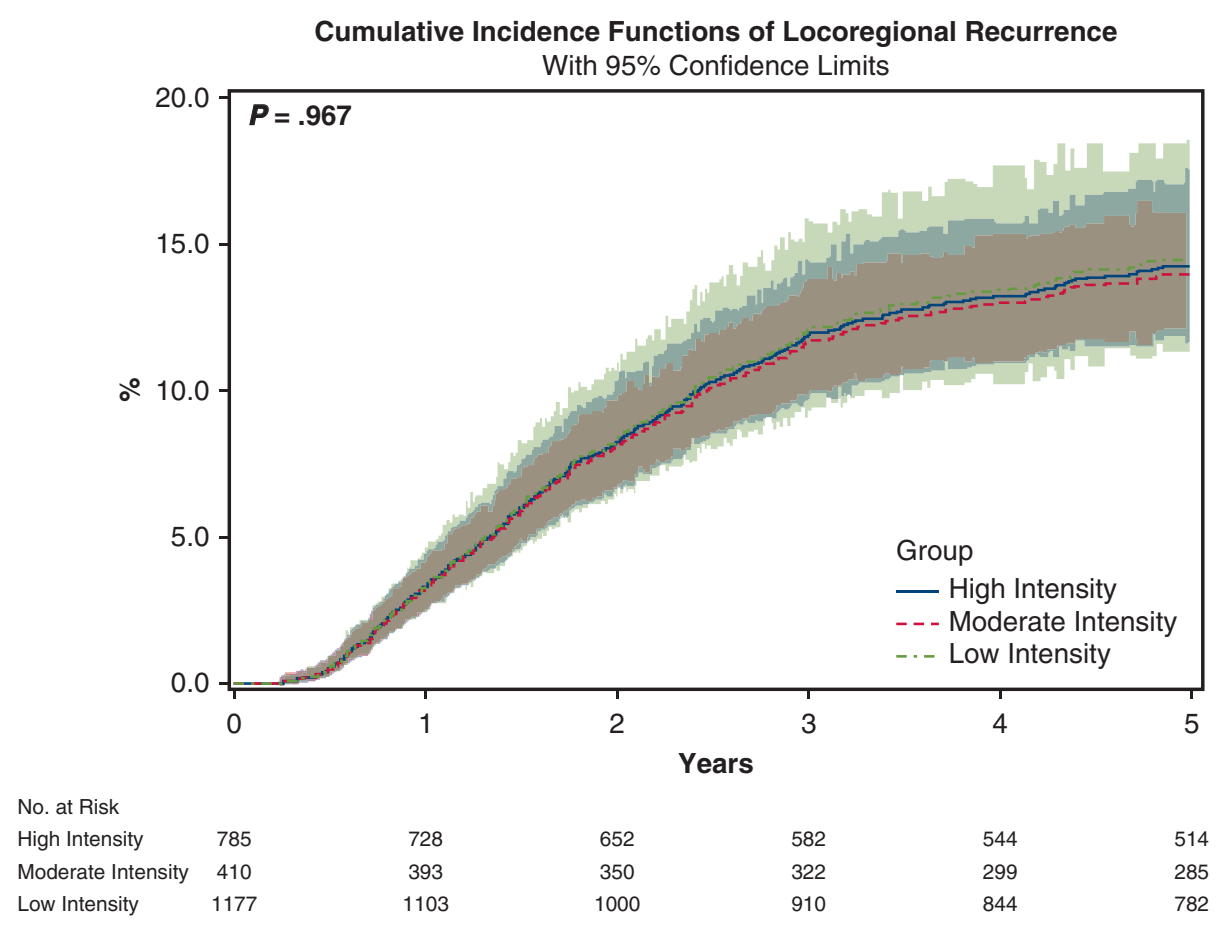

FIGURE 2. Risk of locoregional disease recurrence by surveillance intensity.

Cancer Network, and the International Association for the Study of Lung Cancer offer differing recommendations for intervals and modalities for surveillance. ${ }^{5-7}$ Variability of surveillance in clinical practice is even greater, with adherence to guidelines often being quite poor. In a study of adherence to National Comprehensive Cancer Network and American College of Chest Physicians guidelines, Erb and colleagues ${ }^{13}$ utilized the Surveillance, Epidemiology, and End Results-Medicare database and found that only $61.4 \%$ of patients with stage I NSCLC received guidelineadherent surveillance during the initial 2 years after treatment. ${ }^{13}$ Poor adherence can be partially explained by the paucity of quality longitudinal data to inform best practice.

Using a large, nationally representative database containing complete 5-year follow-up data, we found that surveillance intensity was not associated with overall survival in patients with resected stage I NSCLC. Additionally, surveillance intensity was not associated with differences in time to detection of locoregional recurrence, which represents a group that could potentially benefit from subsequent curative therapy.

Our findings are similar to studies that previously reported on intensity of follow-up. Calman and colleagues ${ }^{3}$ published a meta-analysis of 1669 stage I-III NSCLC patients comparing intensity of postoperative follow-up programs. ${ }^{3}$ The majority of included studies were small and retrospective, with only 1 randomized controlled trial and 1 prospective study. The authors found no difference in OS for NSCLC patients treated with curative intent who received more intensive follow-up. They too did not find a correlation between intensive follow-up and reduced time to local recurrence detection. However, their analysis used wide inclusion criteria, resulting in significant heterogeneity of included studies, patient populations, and surveillance strategies.

In the previously published analysis by members of our study group using special study data to examine the association of CT surveillance intensity in patients with resected stage I-III NSCLC, McMurry and colleagues ${ }^{9}$ observed similar findings. They studied 4463 patients and found that CT surveillance intensity was not associated with overall or postrecurrence survival. However, this analysis highlights the importance of performing stage-specific analysis. There are limited published high quality data that have examined stage-specific surveillance. Homogeneity in surveillance practices and longterm outcomes cannot be assumed across all stages of disease. In fact, McMurry and colleagues ${ }^{9}$ observed that patients with higher stage disease were imaged more frequently. This limits the direct conclusions that can be inferred to the influence of surveillance imaging intensity on patients with early stage disease. In this planned analysis, we exclusively studied patients with stage I NSCLC using independent methodology for classifying and grouping patients into surveillance cohorts. This allowed for comparison within a more homogeneous population, and thus allowed for less biased estimates of disease recurrence and survival. 
TABLE 3. Competing risks model for time to locoregional cancer recurrence

\begin{tabular}{|c|c|c|}
\hline Variable & $\begin{array}{l}\text { Hazard ratio }(95 \% \\
\text { confidence interval) }\end{array}$ & $P$ value \\
\hline Tumor size (mm) & $1.02(1.00-1.03)$ & .014 \\
\hline Substance abuse & $1.67(1.08-2.58)$ & .022 \\
\hline $\begin{array}{l}\text { Resection type } \\
\text { Wedge resection } \\
\text { Segmentectomy } \\
\text { Lobectomy/bilobectomy } \\
\text { Pneumonectomy }\end{array}$ & $\begin{array}{c}\text { Reference } \\
1.30(0.70-2.42) \\
0.55(0.35-0.89) \\
0.77(0.31-1.91)\end{array}$ & .005 \\
\hline $\begin{array}{l}\text { Pathologic stage } \\
\text { 1A } \\
1 \mathrm{~B} \\
1\end{array}$ & $\begin{array}{c}\text { Reference } \\
0.93(0.68-1.27) \\
1.02(0.56-1.85)\end{array}$ & .901 \\
\hline $\begin{array}{l}\text { Histology } \\
\text { Adenocarcinoma } \\
\text { Squamous } \\
\text { Other histology } \\
\text { Unknown }\end{array}$ & $\begin{array}{c}\text { Reference } \\
0.91(0.70-1.19) \\
0.51(0.36-0.74) \\
0.82(0.49-1.37)\end{array}$ & .004 \\
\hline $\begin{array}{l}\text { Number of lymph nodes } \\
\text { sampled }\end{array}$ & $0.68(0.33-1.42)$ & .305 \\
\hline $\begin{array}{l}\text { Surveillance group } \\
\text { Low intensity } \\
\text { Moderate intensity } \\
\text { High intensity }\end{array}$ & $\begin{array}{c}\text { Reference } \\
0.98(0.72-1.35) \\
1.03(0.74-1.43)\end{array}$ & .944 \\
\hline
\end{tabular}

In addition to our focus on patients with stage I NSCLC, this analysis provides unique information on 5-year development risk of locoregional/distant recurrence as well as NPLC, modes of detection of recurrence, and postrecurrence treatment patterns. Theoretically, detection of asymptomatic recurrence or NPLC could offer a survival advantage because these diseases are more likely to be early stage and amenable to therapy. McMurry and colleagues ${ }^{9}$ found that symptomatic presentation of recurrence was associated with worse postrecurrence survival in patients with stage I-III NSCLC. However, asymptomatic presentation is likely a function of the patient's initial stage of disease. Although our results do not support the aggressive use of CT surveillance, this does not preclude the use of CT surveillance for stage I patients. Our data have shown that recurrence and NPLC in stage I patients are often detected at the asymptomatic period, and many go on to receive subsequent therapy. Sixty-three percent of locoregional recurrences and $72 \%$ of NPLC were detected via routine surveillance. Other stage-specific analyses have shown similar findings. Lou and colleagues ${ }^{4}$ retrospectively reviewed patients who had undergone resection for NSCLC and subsequently underwent CT surveillance every 6 to 12 months. They identified 1294 patients with stage I or II disease, of whom $257(20 \%)$ had recurrence. Similar to our study, surveillance CT detected $61 \%$ of asymptomatic recurrences in these patients. Approximately $78 \%$ of the recurrences in early stage patients reported by Lou and colleagues ${ }^{4}$ underwent subsequent therapy, with $12 \%$ being offered surgery with or without radiation. Our study identified that $73 \%$ of locoregional recurrences underwent subsequent therapy, with at least $7 \%$ undergoing surgical treatment.

The role of surveillance imaging has previously been debated given that the efficacy of postrecurrence therapies has not been consistently demonstrated. However, in a large retrospective study of 9001 patients with stage I or III NSCLC with postresection recurrence, subsequent treatment has been shown to be highly beneficial. Using the same special study, Wong and colleagues ${ }^{14}$ compared postrecurrence survival between those who received active therapy versus supportive care. Similar to our study, a high percentage of patients $(79.5 \%)$ with locoregional recurrence received some form of treatment. Median survival for these patients was 19.9 months compared with 4.4 months in patients who received supportive care only. This same pattern was observed in distant recurrence. In our study, $69.4 \%$ of patients with distant recurrence underwent some form of therapy, and more frequent imaging was associated with higher incidence of detection of distant recurrence. Wong and colleagues ${ }^{14}$ reported that $77.3 \%$ of patients with distant metastases underwent treatment, which translated to longer median survival (11.6 months vs 3.0 months).

There are important study limitations to note. Our study consisted of patients that were diagnosed more than 10 years ago. These patients were used so additional data abstraction on 5-year follow-up data could be performed. Thus, there was a trade-off required to capture complete 5-year follow-up information, including recurrence and surveillance, which are not routinely captured in the NCDB. An additional and important limitation includes our categorization of surveillance intensity cohorts. In the real world, patients and physicians often deviate from interval-based regimens (especially as time from primary treatment increases). Previous studies have documented remarkably low adherence to existing guideline-recommended surveillance regimens, approximating $60 \% .{ }^{13}$ Additionally, one must contend with several biases when quantifying surveillance in observational research. First, there is healthy survivor bias-patients who remain on their intended surveillance frequency and do not deviate from their regimens are more likely to be healthy. This would be due to the fact that patients who receive more regimented surveillance scans over time would have to be alive, without recurrence, and without new symptoms or findings on surveillance imaging that prompted a change to more frequent imaging. Alternatively, there is a possibility for an unhealthy survivor bias. Patients with a recurrence, NPLC, or new signs or symptoms could be subject to 


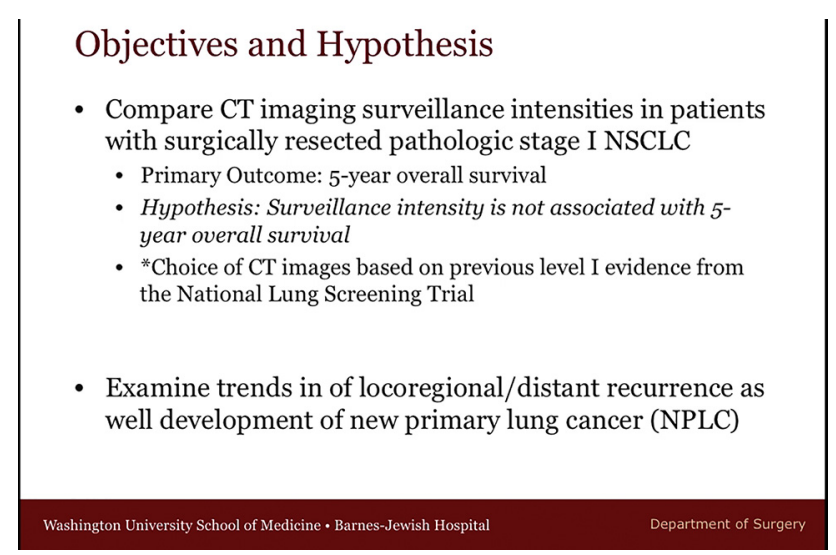

VIDEO 1. Video summary of study design and findings. Video available at: https://www.jtcvs.org/article/S0022-5223(18)32827-7/fulltext.

more frequent imaging, resulting in a higher number of scans. Due to these challenges, there is no perfect method to measuring surveillance intensity in observational studies. Thus, we created our cohorts a priori based on how surveillance initially happened, using time from surgery to first surveillance scan as a proxy. As expected, we found that patients in the dataset did not adhere to strict regimens of surveillance imaging throughout their follow-up. However, the median numbers of scans per person year in each cohort were significantly different between the cohorts. An additional limitation is that our analysis is confined to the variables captured in the NCDB. We observed a trend toward lower-intensity surveillance in patients who did not undergo any therapy for locoregional/distant recurrence. It is possible that clinicians who survey less aggressively tend to not pursue treatment for patients who recur, representing a treatment selection bias that could lead to worse OS. However, our analysis found no difference in OS between the surveillance intensity cohorts. Alternatively, it is possible that these patients had concerning factors that were not captured in our dataset. Factors, including functional status, pulmonary function, margin distance, and presence of lymphovascular invasion are not included in the special study dataset.

Despite these limitations, our study exhibits several strengths. Our study utilized high quality, longitudinal data from reabstracted $\mathrm{NCDB}$ records that contain complete 5 -year recurrence and survival information. The dataset also includes surveillance imaging indications that are critical to distinguish true surveillance from imaging ordered to investigate a new sign or symptom. Additionally, because the NCDB includes $70 \%$ of new lung cancers in the United States, these data are broadly representative and our conclusions are generalizable. ${ }^{15}$

Surveillance intensity was not associated with OS for these patients with stage I disease, suggesting that surveillance is not a 1-size-fits-all approach. Necessary considerations when determining the optimal utilization of any surveillance test include factors such as accuracy, cost and reimbursement for diagnostic studies, the invasiveness of

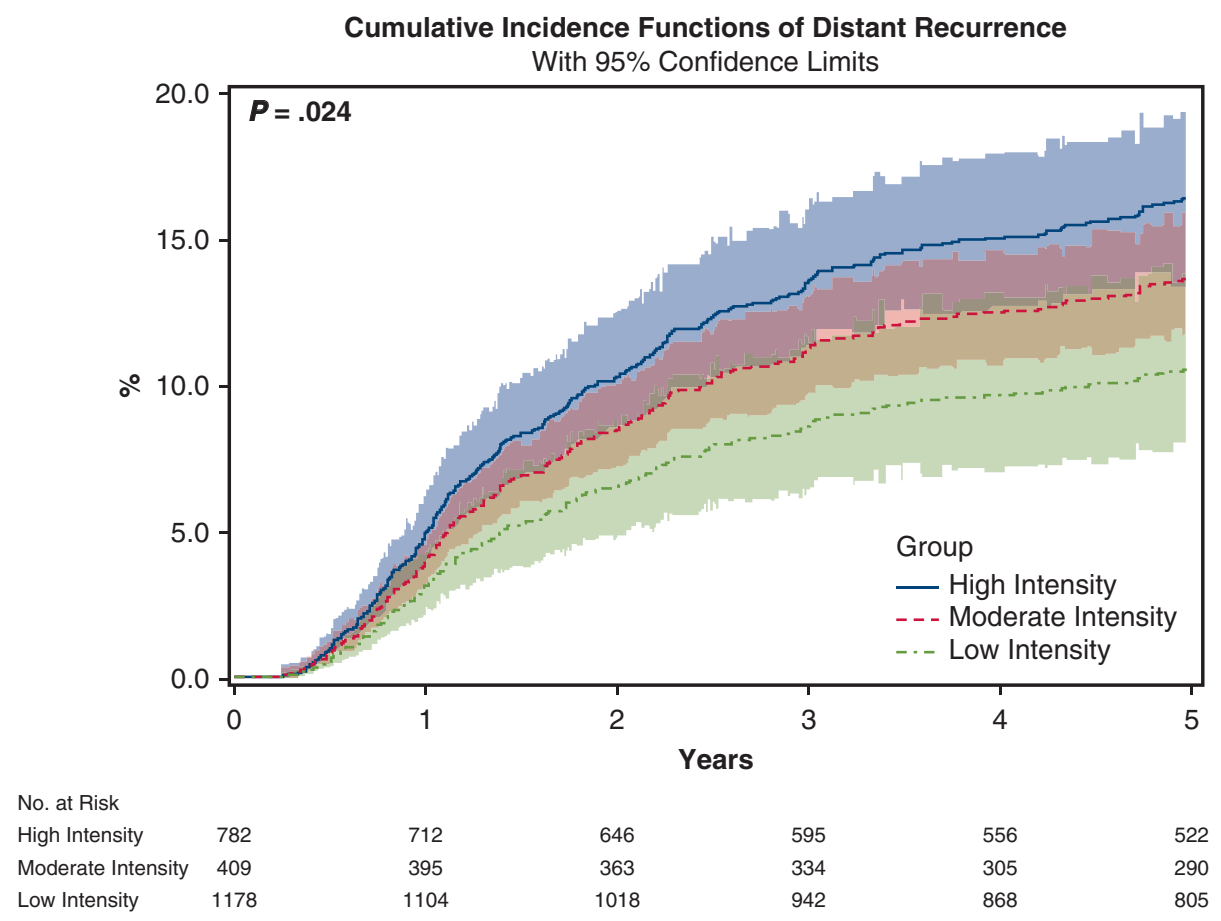

FIGURE 3. Risk of distant recurrence by surveillance intensity. 
TABLE 4. Competing risks model for time to distant cancer recurrence

\begin{tabular}{|c|c|c|}
\hline Variable & $\begin{array}{l}\text { Hazard ratio }(95 \% \\
\text { confidence interval) }\end{array}$ & $P$ value \\
\hline Tumor size (mm) & $1.02(1.01-1.04)$ & .001 \\
\hline $\begin{array}{l}\text { Resection type } \\
\text { Wedge resection } \\
\text { Segmentectomy } \\
\text { Lobectomy/bilobectomy } \\
\text { Pneumonectomy }\end{array}$ & $\begin{array}{c}\text { Reference } \\
1.28(0.63-2.58) \\
0.91(0.63-1.30) \\
1.90(0.90-4.03)\end{array}$ & .121 \\
\hline $\begin{array}{l}\text { Pathologic stage } \\
\text { 1A } \\
\text { 1B } \\
1\end{array}$ & $\begin{array}{c}\text { Reference } \\
0.88(0.64-1.21) \\
1.26(0.68-2.32)\end{array}$ & .504 \\
\hline $\begin{array}{l}\text { Histology } \\
\text { Adenocarcinoma } \\
\text { Squamous } \\
\text { Other histology } \\
\text { Unknown }\end{array}$ & $\begin{array}{c}\text { Reference } \\
0.62(0.46-0.83) \\
0.71(0.49-1.03) \\
1.01(0.64-1.61)\end{array}$ & .006 \\
\hline $\begin{array}{l}\text { Histologic grade } \\
\text { Well differentiated } \\
\text { Moderately differentiated } \\
\text { Poorly differentiated } \\
\text { Undifferentiated } \\
\text { Unknown }\end{array}$ & $\begin{array}{c}\text { Reference } \\
1.72(1.13-2.60) \\
2.01(1.29-3.13) \\
2.73(0.84-8.81) \\
2.18(1.28-3.71)\end{array}$ & .018 \\
\hline $\begin{array}{l}\text { Number of lymph nodes } \\
\text { sampled }\end{array}$ & $1.02(0.79-1.31)$ & .886 \\
\hline $\begin{array}{l}\text { Surveillance group } \\
\text { Low intensity } \\
\text { Moderate intensity } \\
\text { High intensity }\end{array}$ & $\begin{array}{c}\text { Reference } \\
1.31(0.93-1.85) \\
1.59(1.12-2.27)\end{array}$ & .032 \\
\hline
\end{tabular}

Boldface indicates statistical significance $(P<.05)$.

diagnostic procedures, and the emotional stress from fear of recurrence. ${ }^{1,3}$ An optimal, patient-centered approach to surveillance should be driven by knowledge of tumor biology, patient history, patient preference, and candidacy for subsequent treatment. Additional high-quality longitudinal studies are needed to determine how surveillance intensity best fits into this comprehensive approach.

\section{Conflict of Interest Statement}

Authors have nothing to disclose with regard to commercial support.

\section{References}

1. Mollberg NM, Ferguson MK. Postoperative surveillance for non-small cell lung cancer resected with curative intent: developing a patient-centered approach. Ann Thorac Surg. 2013;95:1112-21.

2. Taylor MD, Nagji AS, Bhamidipati CM, Theodaosakis N, Kozower BD, Lau CL, et al. Tumor recurrence after complete resection for non-small cell lung cancer. Ann Thorac Surg. 2012;93:1813-20.

3. Calman L, Beaver K, Hind D, Lorigan P, Roberts C, Lloyd-Jones M. Survival benefits from follow-up of patients with lung cancer: a systematic review and meta-analysis. J Thorac Oncol. 2011;6:1993-2004.

4. Lou F, Sima CS, Rusch VW, Jones DR, Huang J. Differences in patterns of recurrence in early-stage versus locally advanced non-small cell lung cancer. Ann Thorac Surg. 2014;98:1755-60.
5. Rubins J, Unger M, Colice GL, American College of Chest Physicians. Followup and surveillance of the lung cancer patient following curative intent therapy: ACCP evidence-based clinical practice guideline (2nd edition). Chest. 2007 132(3 Suppl):355S-67S.

6. Ettinger D, Wood D, Aisner D, Akerley W, Bauman J, Chirieac LR, et al. Nonsmall cell lung cancer, version 5.2017. NCCN clinical practice guidelines in oncology. J Natl Compr Canc Netw. 2017;15:504-35.

7. Saunders M, Sculier JP, Ball D, Capello M, Furuse K, Goldstraw P, et al Consensus: the follow-up of the treated patient. Lung Cancer. 2003;42(Suppl 1):S17-9.

8. Colt HG, Murgu SD, Korst RJ, Slatore CG, Unger M, Quadrelli S. Follow-up and surveillance of the patient with lung cancer after curative-intent therapy: diagnosis and management of lung cancer, 3rd ed: American College of Chest Physicians evidence-based clinical practice guidelines. Chest. 2013;143:e437S-54S.

9. McMurry TL, Stukenborg GJ, Kessler LG, Colditz GA, Wong ML, Francescatti AB, et al. More frequent surveillance following lung cancer resection is not associated with improved survival: a nationally representative cohort study. Ann Surg. 2018;268:632-9.

10. Aberle DR, Adams AM, Berg CD, Black WC, Clapp JD, Fagerstrom RM, et al Reduced lung-cancer mortality with low-dose computed tomographic screening. N Engl J Med. 2011;365:395-409.

11. Church TR, Black WC, Aberle DR, Berg CD, Clingan KL, Duan F, et al. Results of initial low-dose computed tomographic screening for lung cancer. $N$ Engl J Med. 2013;368:1980-91.

12. Jaklitsch MT, Jacobson FL, Austin JH, Field JK, Jett JR, Keshavjee S, et al. The American Association for Thoracic Surgery guidelines for lung cancer screening using low-dose computed tomography scans for lung cancer survivors and other high-risk groups. J Thorac Cardiovasc Surg. 2012;144:33-8.

13. Erb CT, Su KW, Soulos PR, Tanoue LT, Gross CP. Surveillance practice patterns after curative intent therapy for stage I non-small-cell lung cancer in the Medicare population. Lung Cancer. 2016;99:200-7.

14. Wong ML, McMurry TL, Stukenborg GJ, Francescatti AB, Amato-Martz C, Schumacher JR, et al. Impact of age and comorbidity on treatment of nonsmall cell lung cancer recurrence following complete resection: a nationally representative cohort study. Lung Cancer. 2016;102:108-17.

15. Stewart AK, Bland KI, McGinnis LS, Morrow M, Eyre HJ. Clinical highlights from the national cancer data base, 2000. CA Cancer J Clin. 2000;50:171-83.

Key Words: lung cancer, surveillance, CT scan

\section{Discussion}

Introduction. If we could all start to take our seats, we really need to get moving with the next session. Well, before we begin, I'd like to acknowledge some of our sponsors. Medtronic and Intuitive Surgical as educational supporters of the 2018 meeting, Abbot, Edwards Lifesciences, Medtronic, LivaNova, Intuitive Surgical, and Zimmer Biomed as supporters of breakfast, lunch, and this evening's symposium taking place on Thursday and Friday of the meeting. Don't miss the lunchtime symposium sponsored by Medtronic on meaningful innovations from 12:30-1:45 PM in Salon 6, followed by a residents' symposium on transitioning to practice from 1:45-3:00 PM in the same room. After all the afternoon learning, please join Zimmer Biomed for a reception symposium, "Improving Outcomes and Reducing Complications in Cardiac Surgery" from 3:155:00 PM in Salon 4.

A couple of social notes. The Spanish Nights theme dinner will be this evening and the attire is cocktail. All attendees must have a badge to enter the event, so please make sure that you and your registered family and guests 
wear your badges, and please be sure to sign up for a table for Saturday night's banquet. The seating chart and signup sheets are located in the registration area. As moderator to this session, I want to remind presenting authors that their disclosure slide should precede their presentation and can be displayed for 3 seconds.

Now I'd like to invite our first presenter for the second session to the podium. This is Dr Melanie Subramanian and she will be presenting "Imaging Surveillance for Surgically Resected, Stage I Nonsmall Cell Lung Cancer: Are More Scans Better?'

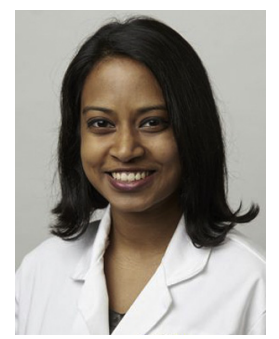

Dr Subramanian. I'd like to thank the Western for allowing us to present our study. There are more than 400,000 estimated lung cancer survivors in the United States, and that number is likely to increase with improvements in therapy as well as lung cancer screening. Patients with early stage, surgically resected, non-small cell lung cancer (NSCLC) remain at risk for development of disease recurrence as well as new primary lung cancer. This makes postoperative surveillance, and especially surveillance imaging, an important component of care for survivorship. However, there are limited high-quality longitudinal data to best inform evidence-based regimens that are comprehensive of both imaging modality as well as intensity. Additionally, previous literature and existing guidelines have traditionally clumped together multiple stages of disease, and it is unclear that these patients should all be managed the same.

When we look at recommendations from national practice organizations like the American College of Chest Physicians, the National Comprehensive Cancer Network, and the International Association for the Study of Lung Cancer, we can see that imaging at intervals varied quite a bit with intervals ranging from every 3 months to annual visits. This heterogeneity is likely reflective of the lack of high quality longitudinal data, and this is particularly true for patients with stage I disease where surgeons are likely to play a lead role in surveillance.

We proposed a study to compare computed tomography (CT) imaging surveillance intensities in patients with surgically resected, pathologic stage I NSCLC. Our primary outcome of interest was 5-year overall survival and we hypothesized that surveillance intensity was not associated with 5-year overall survival. With regard to imaging modality, we chose to focus on CT images given previous level I evidence from the National Lung Screening Trial that suggested the superiority of CT images compared to chest radiograph in lung cancer screening. In addition to our primary objective, we examined trends in locoregional as well as distant disease recurrence and examined trends in the development of new primary lung cancer. We partnered with the American College of Surgeons Commission on Cancer to perform a special study, which allowed for the abstraction of enhanced patient information that is not otherwise obtained in the National Cancer Database. All Commission on Cancer sites that contribute to the National Cancer Database were required to participate. In this special study, the Commission on Cancer collected information on detailed comorbidities, 5-year overall survival, disease recurrence, development of new primary lung cancer, and all postoperative images as well as an indication for each image.

Using the special study data, we performed a retrospective cohort study with 3 surveillance intensity cohorts. These cohorts were based off of time from surgery to the first CT surveillance image and were informed from previously published guidelines. These cohorts were based off of visit windows that mirrored 3 -month, 6-month, and 12-month time points from surgery and they corresponded to high-intensity, moderateintensity, and low-intensity cohorts, respectively.

This is a schematic of the special study mechanism that was used. We started with all commission on cancer sites that contributed to the National Cancer Database and up to 10 patients from each site were randomly selected for data abstraction. These patients were required to have a diagnosis of NSCLC and have undergone curative intent therapy between 2006 and 2007. Additionally, they were required to have complete 5year follow-up information. From there, we imposed additional inclusion and exclusion criteria, identifying patients with pathologic stage I disease and having their first surveillance CT image fall within 1 of our predesignated cohorts. We excluded patients who were symptomatic at the time of their first CT where the indication of the first CT was unknown. From here, we obtained 2565 patients of whom 845,1282 , and 438 patients fell within the high-intensity, moderate-intensity, and low-intensity cohorts, respectively.

For statistical analysis, we performed a mixture of Cox-proportional hazard modeling as well as Kaplan-Meier analysis to model survival. We performed competing risk analysis to examine locoregional as well as distant disease recurrence. Of note, patients who were found to have positive surgical margins on initial analysis were excluded from competing risk analysis.

This table represents demographic information across cohorts. We observed similar age, gender, race representation, and comorbidity profiles across cohorts. However, we should note that high-intensity and moderate-intensity cohorts had a higher prevalence of receiving radiation.

When looking at tumor-related variables, we identified similar surgical resection type across cohorts with lobectomy being the most common procedure. Additionally, 
tumor size, histology type, grade, and positive surgical margin rate were similar across cohorts.

On Cox proportional hazard modeling, we noted that surveillance intensity was not associated with 5-year survival. Variables, including age, gender, comorbidities like chronic obstructive pulmonary disease, congestive heart failure, and psychiatric disease, positive margin status, receipt of radiation therapy, histologic grade, and nonlobectomy resection type were all associated with 5-year survival.

On Kaplan-Meier analysis, we observed similar 5-year overall survival probabilities across cohorts, averaging around $70 \%$. The log rank statistic was 0.37 , suggesting that surveillance intensity was not associated with 5-year overall survival.

When examining trends and locoregional disease recurrence, we identified a locoregional recurrence rate of $13 \%$. Approximately $50 \%$ of locoregional recurrences occurred in the same lung only, whereas $20 \%$ of locoregional recurrences were noted in both the same lung as well as regional lymph nodes. On competing risk analysis, we noted only tumor size to be associated with risk of locoregional recurrence. Surveillance intensity was not significantly associated.

In examining trends of distant disease recurrence, we identified a metastatic rate of $13 \%$. The most common sites of metastases were bone, contralateral lung, and brain in descending order. On competing risk analysis, significant covariates for distant disease recurrence included tumor size; histologic grade; and interestingly, surveillance intensity.

In examining trends of new primary lung cancer, we identified a new primary lung cancer development rate of approximately $7.2 \%$. New primary lung cancer was determined by the treating physician as reported in the medical record. Looking at information regarding new primary lung cancer, approximately $56 \%$ had a different histology than the initial tumor.

There are some important limitations to note to our study. One is the timeliness of the data. Our study included patients who were treated between 2006 and 2007. However, this was to allow for complete 5-year follow-up information to be obtained as well as additional time to allow for the special study to abstract data and subsequent data processing. Additionally, the special study was limited in the fact that there was no collection of subsequent staging information for recurrences in new primary lung cancers.

There are some important strengths to our study to note. First is the fact that we are able to obtain not only the postoperative imaging information, but also the indication for each study. Thus, we had confirmation that these studies were obtained for true surveillance purposes. Additionally, we decided to focus on only stage I
NSCLC because previous studies have combined stage I through III disease and thus we eliminated some of the heterogeneity in the interpretation of our results. An additional strength is that we had relatively complete data of 5-year follow-up information, and finally, because the National Cancer Database captures $70 \%$ of incident lung cancers. The results from our study were fairly generalizable and have real-world clinical application.

So in summary, surveillance intensity is not associated with 5-year overall survival in patients with pathologic stage I, surgically resected disease and our study shows that surveillance intensity should not fit a 1-size-fits all approach. Rather, imaging surveillance should be guided by knowledge of tumor biology, patient history, patient preference, and potential candidacy for subsequent treatment.

I would like to thank the Wash U Thoracic Surgery Clinical Research Group and our funding sources from the Patient Centered Outcomes Research Institute and National Institutes of Health T32 training grants. Thank you.

Moderator. The discussion will be open by David Cooke.

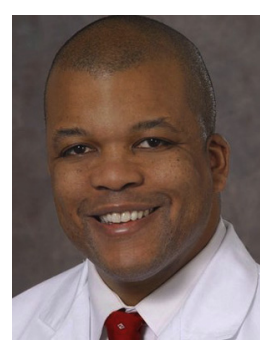

Dr David Cooke (Sacramento, Calif). Thank you. I would like to thank the Association for inviting me and providing me the opportunity to discuss this very interesting manuscript. I would like to thank Dr Subramanian for a wonderful study and also sending the manuscript to me so far in advance I forgot that you sent it. In fact, I was wondering, well, we're getting close to the meeting, where is this manuscript and I said oh, oh wait a minute, let me just search my old e-mails.

Your study highlights that lung cancer survivorship and surveillance is painted with a broad brush, and it's neither patient centered or targeted. It adds to the literature showing that lung cancer survivorship to be improved. In fact, Leah Backus' group showed 4 years ago that in the communitybased practices, lung cancer survivorship surveillance rarely fits published guidelines and is very heterogenic in nature.

You also point out, so I have basically 3 questions, I have basically 3.5 questions. The first, you point out that the American College of Chest Physicians and National Comprehensive Cancer Network guidelines are relatively descriptive where they start off with moderate or medium intensity for the first 2 years, and then move on to high intensity for the last 3 years for 5 years total. In your study, you look at just the first scan and make a definition of cohorts as low, medium, and high, but the reality is, most patients in those cohorts probably cross cohorts and start off at medium intensity and they move on to high intensity. Did 
you, how did you come, how did you make those definitions and cohorts and do you think that makes your data problematic?

Dr Subramanian. So thank you for that question and those comments, and you bring up a very important point. There is previous literature to suggest that adherence to established guidelines, especially interval-based surveillance within the first 2 years is poor and even being cited as low as $60 \%$, so thus, trying to capture different cohorts and different intensities as it happens in the real world remains a significant challenge. So we used this approach expecting for the fact that a lot of these patients would not adhere to interval-based images especially as time from surgery increases. However, after we established our cohort design, we calculated median number of scans per patient year, patient year in this study being not only time into the study, time contributed study or death, but also time until a new abnormal imaging finding was found because in the real world, if a recurrence of primary lung cancer is detected, the frequency of imaging may change afterward. And based on our calculations, we did find significant differences across cohorts that corresponded to our predesignated cohort intensity definitions. So although our cohorts don't follow exactly every 3 months, every 6 months, or annually, we did note a significant pattern toward that the cohorts were actually truly different.

Dr Cooke. Okay, thank you. The second question. Can you just help me understand the role of radiation in the outcomes of your study? Radiation, from my understanding, is an independent predictor for 5-year mortality. Are you controlling radiation for positive surgical margin? Who are these patients, are they, are these patients that receive radiation for a positive margin or are they patients who were initially treated with either stereotactic body radiation therapy or definitive radiation and this is a salvage resection for in-field recurrence?

Dr Subramanian. So within our data, within the National Cancer Database, it was difficult to interpret the true intent for radiation therapy. We thought initially it might be due to surgical positive margins. However, we observed an average about 3\% positive margin rate with about $7 \%$ receiving radiation therapy, so that can only account for a portion of those patients. So we speculated, perhaps it might be due to proximity to the margin or near margins, but we're limited in our data in that we can't speculate, we can't obtain the actual data to determine why these people had radiation treatment.

Dr Cooke. Okay. And then the final question I have, your Kaplan-Meier curves were very compelling that shows no survival difference between low-, median-, and high-intensity screening. What I don't find is a good subanalysis description. So for instance, you find that histologic grade and extent of resection are predictors for mortality, for 5-year mortality. So presumably there's a population of patients who would benefit from the high-intensity surveillance. Did you take, say, your medium high-grade and undifferentiated grade patients, see them as an individual cohort, and then look at low-, medium-, and high-intensity surveillance in that population to see if you see a survival difference. And then a follow-up of this, obviously not designed for this study but for future studies, have you thought about taking those identified patients, so specifically grade, resection, extension, and even margin, and creating a model or a tool to use as a predictor of who would need high-intensity screening and then validate that tool either by going back to the National Cancer Database or using your own robust patient population retrospectively at Wash U?

Dr Subramanian. With regard to your first question of whether or not we performed a subgroup analysis for the Kaplan-Meier analysis, we did not, I think that would be a good future direction to take with this work or potentially in revisions. However, that's why we also looked at Cox proportional hazard modeling to adjust for factors like histology and grade, and even after adjustment we found that surveillance intensity was not associated with 5-year overall survival but your point is well taken, and we should definitely do that subgroup analysis and with regard to the future directions of potentially developing a risk prediction model, I think that's a great idea, especially because the dataset provided from this special study is so unique, we should take advantage of it.

Dr Cooke. Great. Thank you for a wonderful study.

Dr Subramanian. Thank you.

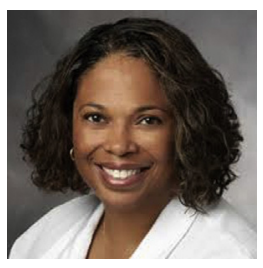

Dr Leah Backhus (Stanford, Calif). Thank you for your presentation, I think it's really timely. Obviously, it's a subject very near and dear to my heart and you bring up the point that we kind of have this 1-size-fits-all approach, which is probably not best. Recently, I think it was last year, the French had their biggest prospective randomized trial looking at this very thing and they concluded the same thing that you concluded here, which is that, you know, high intensity is really of no benefit. If there is any benefit, it really doesn't show up until after 2 years. Did you see any stratification like that in terms of when there might be some benefit, or how it is that you can take your conclusions and go from there toward actually making better recommendations. My second question is with regard to the downsides of high intensity screening. If anything, your survival curves show that those guys didn't do as well although it wasn't significantly so. So did you do any analyses to look at the unintended consequences of doing more aggressive screening, for instance, radiation oncologists tend to do very aggressive surveillance where they do positron emission tomography/computed tomography every 3 months almost which is, in most people's opinion, excessive. Thank you. 
Dr Subramanian. With regard to your first question, did we examine by time if there were any changes in survival or when higher intensity surveillance might be useful. We didn't divide into specific time points like pre- or post2 years, for example, and most guidelines suggest changing surveillance intensity. However, I think that would be a good future analysis to do. And with regard to the second question, which I believe was potential harms of higher surveillance intensity. We were able to capture information on whether or not patients underwent treatment or had a biopsy that was performed, and I think probably one of the most useful pieces of information would be to see whether or not there were high false positive rates associated with more frequent imaging. However, we weren't able to, with the information captured by the special study, to calculate false positive rates but I think for any future study, I think that would be among the most useful pieces of information to obtain.

Dr Backhus. Thank you.

Speaker. That was a great presentation and great study. I just have 1 comment. It's a really big challenge trying to establish a relationship between a diagnostic workup and long-term outcomes in the observational setting. I have great empathy for those challenges because our team is facing those same challenges, studying incidentally detected lung nodules in the observational setting. That said, I think you have a superb research team and you guys have done the best you can. I also want to point out that there was a trial that was presented at the European Society for Medical Oncology meeting in 2017, it's a French trial and they randomized patients to either examination and a radiograph versus examination and computed tomography in a heterogeneous group of lung cancer patients and there were no differences in 3-year disease-free survival and there were no differences in 8-year overall survival. That paper hasn't been published as far as I can tell, I think it still has to be critiqued and we have to look into the details of the study, but it may be that there really is no relationship between surveillance and long-term outcomes although that's contrary to our belief. So anyway, I just wanted to point that out and congratulations on a great study.

Dr Subramanian. Thank you. 
9866 patients with surgically

resected pathologic stage I-III NSCLC identified by Special Study

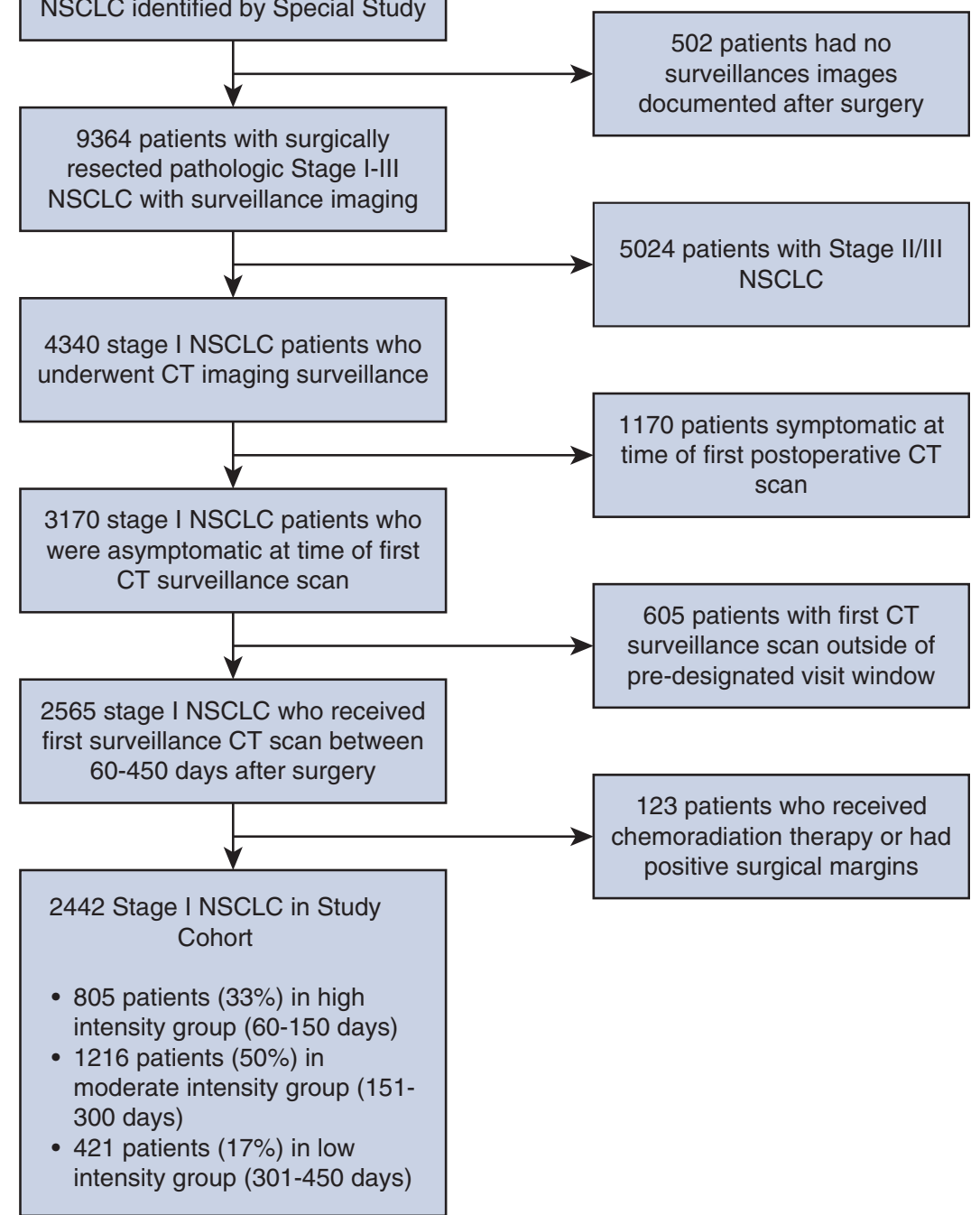

FIGURE E1. Inclusion/exclusion criteria and selection of study cohort. NPLC, New primary lung cancer; $C T$, computed tomography. 
TABLE E1. Method of detection: Recurrence and new primary lung cancer (NPLC)

\begin{tabular}{|c|c|c|c|}
\hline Method of detection & $\begin{array}{c}\text { Locoregional } \\
\text { recurrence }(n=333)\end{array}$ & $\begin{array}{l}\text { Distant recurrence } \\
\qquad(\mathbf{n}=\mathbf{3 3 3})\end{array}$ & $\begin{array}{c}\text { NPLC } \\
(n=178)\end{array}$ \\
\hline $\begin{array}{l}\text { Finding detected on nonroutine clinician visit, prompted by patient-detected } \\
\text { sign/symptom }\end{array}$ & $65(19.5)$ & $135(40.5)$ & $45(25.3)$ \\
\hline Finding detected on routine visit, physician-detected new sign & $20(6.0)$ & $10(3.0)$ & - \\
\hline Finding detected on routine surveillance imaging, patient asymptomatic & $210(63.1)$ & $122(36.6)$ & $128(71.9)$ \\
\hline Finding detected as part of workup for other local/distant/NPLC disease & $22(6.6)$ & $45(13.5)$ & - \\
\hline Finding incidentally detected for unrelated/other imaging & $3(0.9)$ & $15(4.5)$ & - \\
\hline Unable to determine & $13(3.9)$ & $6(1.8)$ & $5(2.8)$ \\
\hline
\end{tabular}

Values are presented as $\mathrm{n}(\%)$. NPLC, New primary lung cancer.

TABLE E2. Subsequent treatment of locoregional/distant recurrence

\begin{tabular}{lcc}
\hline \multicolumn{1}{c}{ Treatment } & $\begin{array}{c}\text { Locoregional } \\
\text { recurrence }(\mathbf{n}=\mathbf{3 3 3})\end{array}$ & $\begin{array}{c}\text { Distant } \\
\text { recurrence }(\mathbf{n}=\mathbf{3 3 3})\end{array}$ \\
\hline No treatment & $70(21.0)$ & $83(24.9)$ \\
Surgery only & $21(6.3)$ & $14(4.2)$ \\
Chemotherapy only & $88(26.4)$ & $79(23.7)$ \\
Radiation therapy only & $52(15.6)$ & $70(21.0)$ \\
Combination therapy & $82(24.6)$ & $68(20.4)$ \\
Unable to determine & $20(6.0)$ & $19(5.7)$ \\
\hline
\end{tabular}

Values are presented as $\mathrm{n}(\%)$ 